\section{GEMEINSAME THERAPIEENTSCHEIDUNG}

\section{Patienten tun sich schwer, dem Arzt zu widersprechen}

Idealerweise sollten Patienten und Ärzte gemeinsam zu einer Therapieentscheidung finden. Dies scheitert jedoch häufig an der Unfähigkeit von Patienten, Nein zu sagen, ergab eine internetbasierten Studie zum Thema Shared Decision Making mit 1340 Patienten im Alter von 40 bis 60 Jahren. Im konkreten Fall ging es um die Behandlung eines in der Publikation nicht näher spezifizierten Herzleidens, bei dem es mehrere Handlungsoptionen, darunter aber keine eindeutig korrekte, gab. Die Studienteilnehmer hatten im Durchschnitt einen hohen Bildungsstand. Zur gemeinsamen Ent- scheidungsfindung bekannten sich fast $70 \%$. Schlüsselte man aber die patientenseitigen Elemente des gemeinsamen Entscheidens auf, gaben $93,1 \%$ an, sie würden dem Arzt Fragen stellen, und 94\% wollten ihre Präferenzen besprechen. Aber nur 14\% würden ihre Ablehnung ausdrücken, sollten sie nicht mit den Ratschlägen des Arztes übereinstimmen. Ihren Ärzten zu widersprechen fällt offenbar den meisten Patienten schwer - viel schwerer, als Fragen zu stellen oder Wünsche zu äußern.

JAMA 2012, online 9. Juli; doi:10.1001/archinternmed.2012.2360

\title{
NÜTZLICHE NEBENWIRKUNG
}

\section{ACE-Hemmer schützen vor Pneumonie}

Die Verstärkung des Hustenreizes gilt als Manko der ACE-Hemmung. Dabei scheint sie auch ihr Gutes zu haben: Patienten unter ACE-Hemmer-Therapie entwickeln einer Metaanalyse zufolge etwa ein Drittel weniger Pneumonien. Möglicherweise schützt die unter ACE-Hemmung leichtere Auslösbarkeit des Hustenreflexes die Atemwege vor Keimen aus dem Oropharynx. Für die Metaanalyse wurden 37 Studien herangezogen, in denen ACE-Hemmer bzw. $\mathrm{AT}_{1}$ Antagonisten entweder mit einer Kontroll- therapie oder direkt miteinander verglichen worden waren. Unter ACE-hemmender Therapie lag die Pneumonierate um $34 \%$ niedriger als unter einer Kontrolltherapie und um 37\% niedriger als unter Sartanen. Die Schutzwirkung der ACEHemmer war besonders ausgeprägt bei Patienten, die wegen eines Schlaganfalls oder einer Herzinsuffizienz ein erhöhtes Pneumonierisiko hatten (relative Risikoreduktion um 54\% bzw. 37\%).

BMJ 2012; 345: e4260; doi: 10.1136/bmj.e4260

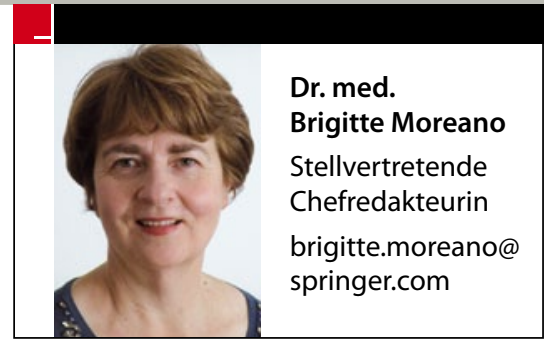

RISIKOFAKTOR FÜR HYPERTONIE

\section{Schlafdefizit lässt den Blutdruck steigen}

Wer werktags weniger als fünf Stunden Schlaf erwischt, hat ein deutlich erhöhtes Risiko, an Bluthochdruck zu erkranken, zeigte eine Studie der Pariser Sorbonne mit 1046 Hausarzt-Patienten im Alter von 40 bis 71 Jahren. Ihr Blutdruck wurde gemessen und ihre nächtliche Schlafdauer erfragt. $51 \%$ der Probanden mit höchstens fünf Stunden Schlaf hatten eine Hypertonie, während dies nur bei 31\% der Personen zutraf, die nachts sieben Stunden schliefen. Vermutlich, so die Autoren, aktiviert ein Schlafdefizit die Hypothalamus-Hypophysen-Nebennierenrinden-Achse. Durch die Vermittlung von Kortisol könnte die cholinerge Vasodilatation gehemmt und die Aktivität von Mineralokortikoiden erhöht werden. Beides steigert den Blutdruck.

J Hypertens 2012; 30:1354-1363

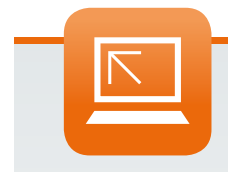

Expertenrat auf springermedizin.de

Fachkundigen Rat in allen medizinischen Fragen erhalten Sie online unter www.springermedizin.de/expertenraete - Die Experten der MMW Prof. Füeßl und Dr. Stiefelhagen beantworten Ihre Fragen auf www.springermedizin.de/ mmw-sprechstunde 